Education Act prompted him to take a position with Representative Major R. Owens (D-NY), Ranking Minority Member of the Committee on Education and the Workforce's Subcommittee on Workforce Protections; Robert Wood Johnson Health Policy Fellow Dr. David A. Pollack, medical director for Mental Health Services West, Inc. of Portland, Oregon, who obtained a position with Senator Edward Kennedy (D-MA) on the Senate Committee on Labor, which will be working on health policy legislation; Foreign Affairs Fellow Susan Rzemien, an international economist with the Treasury Department's Office of Foreign Exchange Operations, who accepted a staff assignment with Senator Richard Lugar (R-IN); the CIA's Donna Counterman, who will work for Representative Christopher Shays (R-CT); APSA-MCI Congressional Fellow Andrew Souvall, a producer with KUTV Channel 2 in Salt Lake City, who joined the staff of Florida Republican Senator Bob Graham; and the State Department's John McNamara, who took a position with Massachusetts Democrat Senator Robert Kennedy.
To supplement what promises to be a very unique year as congressional staff, Fellows will be attending the Wilson Continuing Seminar Series at the Library of Congress, Upcoming speakers include Washington Post correspondent and columnist Dave Broder; William and Mary political scientist and former Fellow Larry Evans; the Honorable Charlie Johnson, House Parliamentarian; and representatives of the Congressional Budget Office, General Accounting Office, and Office of the House Legislative Counsel.

\title{
Southern Illinois University Hosts Fifth Summer Institute
}

\author{
John Foster, Southern Illinois University, Carbondale
}

The 1998 USIA Summer Institute in the American Political System, held from June 26 to August 8, was the fifth on the campus of Southern Illinois University, Carbondale (SIUC). Each participant in the 1998 SIUC institute has responsibility for university-level course on American government, politics, and history in his or her home country, but had little or no previous direct exposure to the United States. Two of the 1998 participants came from Latin America, six from Africa, seven from Asia, and three from the former Soviet Union or Soviet bloc. The program is funded by the United States Information Agency.

The broad goal of this institute is to provide the participants with as thorough an immersion in American government, politics, and political culture as possible. The broad organizing theme of the 1998 institute was the American federal system, with its rather unique division of governmental responsibilities across national, state, and local units. Outside visitors seldom appreciate either the historical role state and local units of government play in the United States or the increased importance of these units in recent years. A secondary theme was to compare and contrast the American approach to the universal problems of governing with the approaches of other nations. Most of the 1998 participants came from countries that have moved either from single-party communist or military rule to fledgling democracies within the past decade. The American experience of over 200 years under the same constitutional form of government provides a sharp contrast with the participants' personal experiences of government.

The institute tried to mix contemporary political science scholarship (including the latest teaching materials for American government) with direct contact with practitioners at all three levels of government. Most of the faculty in SIUC's department of political science as well as a number of faculty from other departments in the College of Liberal Arts and School of Law participated. Also included in the program were two members of the staff of the SIU Public Policy Institute-former U.S. Senator Paul Simon and Mike Lawrence, former press secretary for Governor Jim Edgar.

Classroom time was supplemented with a one-week trip to the Illinois state capital in Springfield and a variety of sites in Chicago and a second week-long trip to Washington, DC. The week in DC included a visit to the headquarters of the American Political Science Association, where all participants received complimentary one-year APSA memberships. The program included a number of single- and partial-day

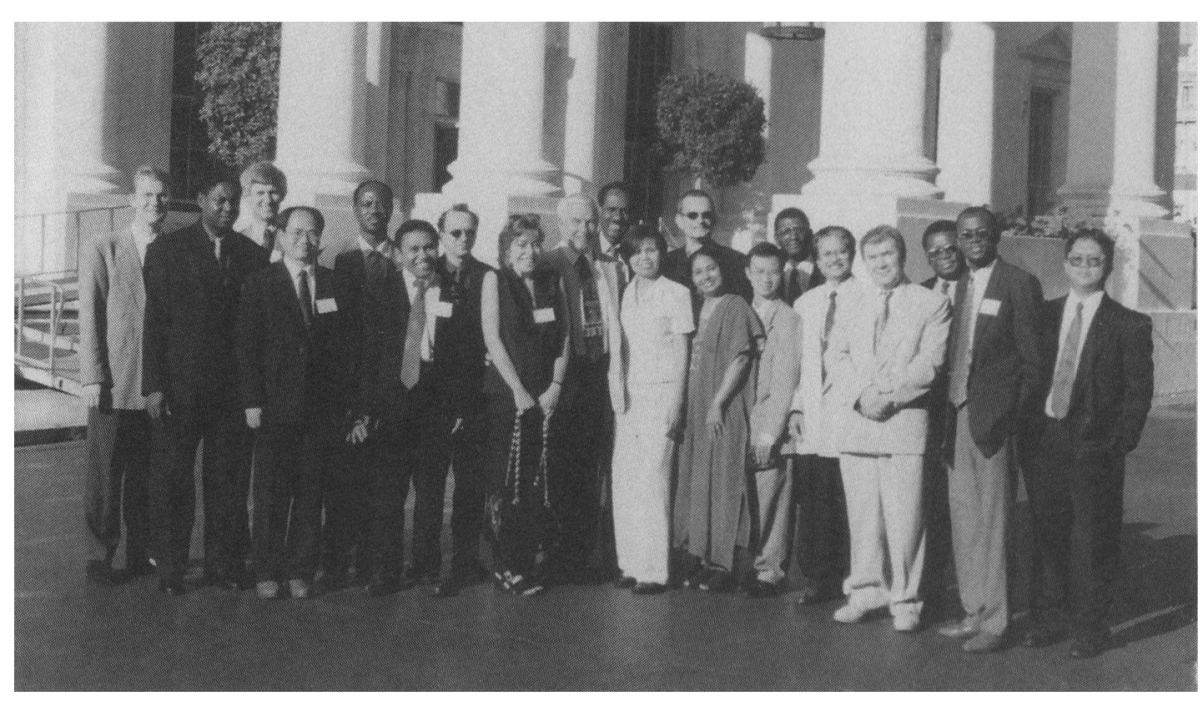

Members of the 1998 USIA Summer Institute gather for a group photo outside the White House. 


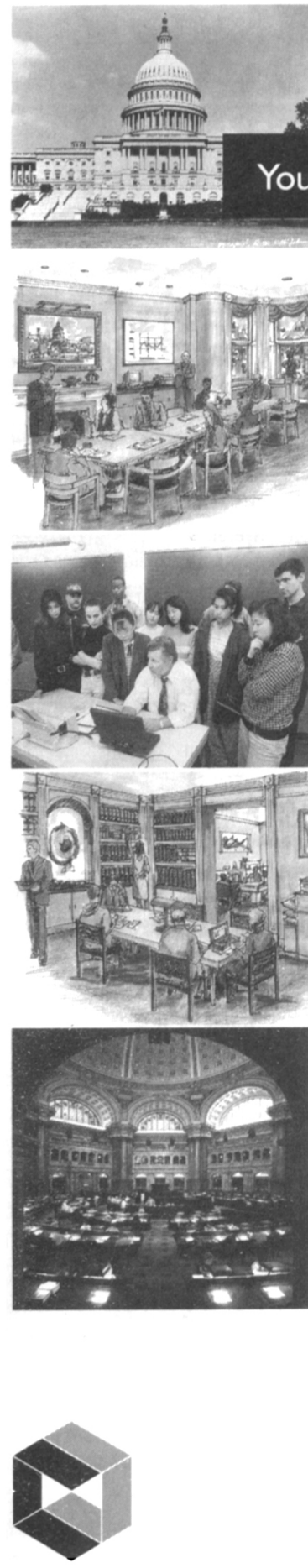

For more information contact:

American Political Science Association 1527 New Hampshire Avenue, NW Washington, DC 20036-1206

Robert J-P. Hauck

Campaign Director

Tel: 202.483 .2512

Fax: 202.482.2657

Email: future@apsanet.org

\section{Your gifts are helping to celebrate the Past by investing in the Future!}

\section{Campaign Goal: $\$ 3$ million to endow professional opportunity grants and the Centennial Center for Political Science}

APSA gratefully acknowledges the support of these contributors to the Centennial Campaign as of February 4, 1999.

Centensial Circle
$\$ 25,000$ or more
Walter E. Beach - C, A, B
Doris Graber
Pendleton Herring
Huang Hsing Foundation \& Chun-tu Hsueh
University of Louisville, in honor of
Aaron Wildarsky
Elinor \& Vincent Ostrom, in honor of
Alma Ostrom \& Leah Hopkins Awan
Estate of Leo A. Shifrin
Frank J. Sorauf

1903 Circle

$\$ 15,000$ or more

Richard F. Fenno, Jr.

Norman Nie

Jack W. Peltason - C, A

\section{Circle}

$\$ 10,000$ or more

Ruth S. Jones \& Warren E. Miller - C, M, A Nannerl \& Robert O. Keohane

Arend Lijphart

Dale Rogers Marshall

\section{Founders' Ciscle}

$\$ \$, 000$ or more

Lucius J. Barker

Robert H. Bates

Susan C. Bourque

David Brady

Roger H. \& Nancy Davidson - C, A, S

Jo Freeman - W

James L. Gibson

Betty Glad - P

John Mark Hansen

Martin 0. Heisler - A

Jennifer P. Hochschild \& C. Anthony Broh

Marthew Holden, Jr.

Gary C. Jacobson
Joyce K. Kallgren

Mary F. Katzenstein \& Peter Katzenstein

Martha Joynt Kumar, in honor of

Ann Devroy - $P$

Norman J. Ornstein

Pi Sigma Alpha

Lucian W. Pye

J. Austin \& Nancy Ranney

Catherine E. Rudder - $C, A, M, B, B r, W$

Paul M. Sniderman

James Q. Wilson

Nancy H. Zingale

\section{Builders' Circle \\ $\$ 2,500$ or more}

David Adamany
James E. Anderson

Anonymous - B

William I. Bacchus

Michoel A. Boer

Barbara B. Bardes

Paul Allen Beck - C, A

John F. Bibby

Charles S. Bullock, III

Philip E. Converse

William J. Daniels

Christopher J. Deering - C, S

Jorge I. Dominguez

Lowrence C. Dodd, in honor of the

Department of Political Science,

University of Minnesota

Leon D. Epstein

Heinz Eulau - C, A

Luis Ricardo Fraga

Kathleen A. Frankovic

Virginia H Gray - C, A

Joel B. Grossman

Charles D. Hadley - C, A

Robert J-P. Hauck - C, A, M

Anne Hopkins

Robert Huckfeldt

M. Kent Jennings

Loch K. Johnson

Charles O. Jones - C, A, $P$

William J. Keefe

Rita Mae Kelly - B
Herbert P. Kitschelt

Allon Kornberg

Kay Lawson

Avery Leiserson

Burdett A. Loomis

Susan A. MacManus - C, A

David B. Magleby - S

Thomas E. Mann

Joel Margolis - $S$

David R. Mayhew

Daniel A. Mazmanian

Paula D. McClain

Cynthia McClintock

Lorraine M. McDonnell \&

M. Stephen Weatherford - $P$

Karen $\mathrm{O}^{\prime}$ Connor

David Scott Palmer, in memory of

Eldon "Bud" Kenworthy

Leonard Parkinson

Michael B. Preston

George H. Quester

Beryl A. Radin

Randall Ripley

Alan Rosenthal

Kay Lehman Schlozman - C, B

Mildred A. Schwartz

Lee Sigelman

Howard J. Silver

Borbara Sindair

Raphael J. Sonenshein

Harvey Starr

Dorothy McBride Stetson

Joseph Stewart, Jr.

Judith Hicks Stiehm - C, A

Clarence N. Sione

Edward Thompson, III, in honor of

Marguerite Ross Barnett \&

Vincent J. Browne - B

James A. Thurber

David B. Truman

Sidney Verba

John C. Wahlke - C, A, P

Charls E. Walker

Kenneth N. Woltz

Paul J. Weber

Susan Welch

David Wilstord, in honor of

Worren Miller - C, M

$\mathrm{C}=$ General Compoign Fund, $\mathrm{A}=$ Artinian Fund, $\mathrm{B}=$ Barnett Fund, $\mathrm{Br}=\mathrm{Bryc \theta}$ Fund, $M=$ Miller Fund, $P=$ Presidency Research Fund, $W=$ Women \& Politics Fund 


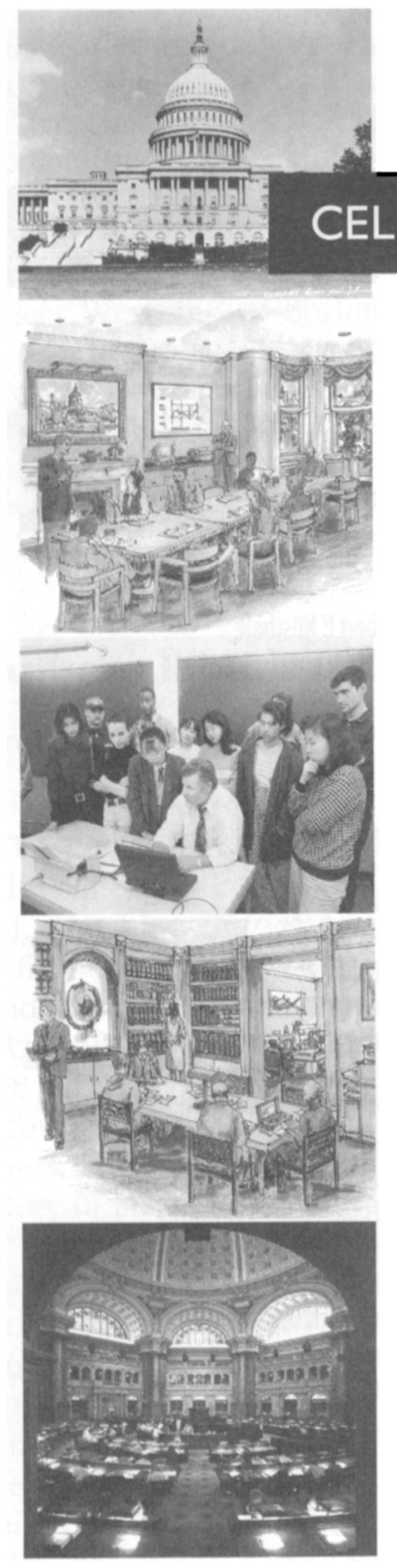

\section{KEY}

$C=$ General Campaign Fund

$A=$ Artinian Fund,

$B=$ Barnett Fund,

$\mathrm{Br}=$ Bryce Fund,

$M=$ Miller Fund,

$\mathbf{P}=$ Presidency Research Fund,

$W=$ Women \& Politics Fund

\section{AMERICAN POLITICAL SCIENCE ASSOCIATION \\ Centennial Campaign}

\section{CELEBRATING THE PAST....}

\section{Second Century Society}

$\$ 1,000$ or more

Anthony Affigne - B

Herbert Alexander - $A, M$

Hayward R. Alker

Gabriel Almond

Patricia Artinian - A

Herbert B. Asher

Samuel H. Barnes - A, M

Jeffrey M. Berry

James W. Bjorkman

Christopher J. Bosso, in honor of the

Political Science faculty at the

University of Pittsburgh

Steven J. Brams

Michael A. Brintnall

James MacGregor Burns

Lief $\mathrm{H}$. Carter

Leonard Champney - A

Clarke E. Cochran

Thomos E. Cronin - C, P

Milton C. Cummings, Jr.

Robert A. Dah

Linda Fowler

Jurg Martin Gabriel

Louis C. Gawthrop - A

Susan W. Hammond

Roderick P. Hart

C. Randall Henning

Mary A. Hepburn, in honor of

Sheilah Mann

Robert J. Huckshorn

William Hudson - A

Karen M. Hult - $P$

Dennis Ippolito

Bruce W. Jentleson

Nolan E. Jones - B

Gerhard Loewenberg

William Mishler, in honor of

Allan Kornberg - $C, A, S$

Janet M. Martin - $P$

John S. Odell

Kenneth T. Palmer

Desiree Pedescleoux - A

Nelson W. Polsby - A

Henry Pratt, in honor of David Truman

David E. Price

Thomas F. Remington

Leroy N. Rieselbach - S

Donna E. Shalala - B

W. Phillips Shively - M

Roberta S. Sigel - C, B

Elliot E. Slotnick, in honor of

Frank J. Sorouf
Thomas A. Spragens, Jr.

Janet D. Steiger - $S$

Sybil L. Stokes

Gerald H. Stollman

Deborah Stone - C, B

Raymond Tatalovich - P

Charles E. Walcott - $P$

Raymond F. Wolfinger, in honor of

Warren E. Miller - M

Maurice C. Woodard

James V. Young

Joseph F. Zimmerman

\section{Ansivessary Society}

$\$ 500$ or more

Joel D. Aberbach, in honor of Robert Lane

Martha Ackelsberg - B

Association of Chinese Political Studies

Henry Bienen

Ann O'Meara Bowman

Emmett Buell - A

Bruce Bueno de Mequito

David A. Caputo

David Collier \& Ruth Berins Collier

M. Margaret Conway

Gary W. Cox

C. Jeremy Curtoys

Vincent \& Anne Dovis, in honor of Charles 0 . Jones

George C. Edwards, III - P

John B. Gates, in memory of Richard Sinopoli

Harvey Glickman, in memory of H. Hubert Wilson

Robert T. Golembiewski

Robert C. Grady - A

Melinda Gann Hall - A, B

John R. Hibbing

Kim Quaile Hill - M

Harry N. Hirsch

John W. Holcombe, in memory of George S. Blair

Robert P. \& Dixie S. Huefner, in honor of Edward Banfield

Patricia A. Hurley - $M$

Peter Juviler - A

Miles Kahler

Samuel A. Kirkpatrick - M

Margaret Levi

Mark Lichbach

David L. Lowery
L. Sandy Maisel, in honor of Worren E. Miller - $M$

J. Donald Moon

Robert Montioy

Robert J. Mundt

Richard W. Murray

Richard E. Neustadt - P

Pippa Norris - M

Joseph S. Nye, Jr.

Carole Pateman - A

Mark P. Petrocca, in honor of Gina \& Joseph Petracca

Gerald M. Pomper - A

Jewel L. Prestage - B

Robert D. Putnam

Donald L. Robinson

Bert A. Rockman - A

Lloyd I. \& Susanne Hoeber Rudolph

Virginia Sapiro

Robert Y. Shopiro - P

Beth Simmons

Harold Stanley - A

Randall Strahan

Jeffrey K. Tulis - $P$

Stephen L. Wasby - A, B, M, S, in memory of Morris Udall

Marcia Lynn Whicker, in honor of Malcolm Jewell - A

Frederick M. Wirt - B

Kenneth K. Wong

Diel S. Wright

David Vogel

Campaigen Friends

$\$ 250$ or more

J. Theodore Anagnoson

Donald G. Baimer

John C. Berg - A

Goyle Binion

Mary Anne Borrelli - P

Randall L. Calvert

David T. Canon - $S$

Cary R. Covington - $P$

Russell Dalton - A

John G. Geer - M

Joyce Gelb - C, B

John W. Harbeson

Robert A. Heineman - A

Mariorie R. Hershey - A

Allen D. Hertzke

Raymond F. Hopkins 


\section{Centennial Camp anign}

BY INVESTING IN THE FUTURE.

E. Terrence Jones - $M$

Mark P. Jones - $M$

William R. Keech - A

Frank Kessler - $P$

John Kinceid

Samuel Krislov \& Judith Gillespie - A

David A. Lake

David D. Laitin

Andrew S. McFarland - A

Eugene Meehan - A

Bruce Miroff - A

New England Political Science Association - A

Susan S. Northeutt

James L. O'Sullivon - Br

Don Racheter

David M. Rayside

Richard \& Rosemary Rose - A

Herbert J. Rubin - A

Arlene W. Saxonhouse

W. Rand Smith

Carol M. Swain - C, A, B

Norman C. Thomas - $P$

Shirley Anne Worshaw - $P$

M. Crawford Young

\section{Campaign Ansociates $\$ 100-\$ 249$}

Shariff Ahmad - S

Gar Alperovitz

Asher Arion - A

Harold F. Bass, Jr. - C, A

David R. Bermon

Thomas M. Collaghy

Colin Campbell - A

Jeffrey E. Cohen - $P$

William J. Crotty

I.M. Destler - A

Donna Robinson Divine

Michael W. Doyle \& Amy Gutmann

Molcolm Feeley - A

Poul Ferber

Michael Genovese - P

Emily R. Gill - B

Joonne Gowo

Fred I. Greenstein - C, P

Joseph M. Grieco

Paul S. Herrnson - A

Kenneth Hoover, in memory of

Edward Artinion - A

Serge Hurtig - C, M

Ronald Kohn

Ellis Katz

Donald F. Kettl - A

John J. Kirlin
Susan S. Lederman - A

Michael H. Levin -S

Michael Lienesch

Roderick MacFarquhar

Harvey C. Manstield, Jr. - A

Scott L. McLean - A

Wilson Corey McWilliams - A

Gabriel Miller - A

Harris N. Miller - S

Charles C. Moskos, Jr.

Elizabeth F. Moulds

Corol Nechemias

David Newman - A

Joseph L. Nogee

Helmut Norpoth - A

Samuel C. Patterson - A

Thomas E. Patterson - A

William D. Pederson

John C. Pierce - M

Dianne Pinderhughes - B

Jorgen Rasmussen - A

APSA Organized Section on

Representation \& Electoral Systems

Nancy L. Rosenblum

Frances M. Rosenbluth

Margaret Ann Rosenthal - A

Anne L. Schneider - B

Byron E. Shafer - A

Kenneth A. Shepsle - M

Steven A. Shull - $P$

Sheldon W. Simon - M

Peter N. Skerry

Robert J. Spitzer - A

Kothleen Tapport - B

Leslie Paul Thiele - A

Kenneth D. Wold - A

Herbert Waltzer - A

Robert E. Ward

Stephen White - A

Clyde Wilcox - A

Robert C. Wood - A

Alan Zuckerman - A

Karen J. Alter

Donald C. Baumer

Seyla Benhabib

Frederic A. Bergerson

Thomos E. Borcherding

Evelyn Z. Brokin

Frances Burke - A

Brenda W. Carter - A

\section{Contributars}

$\$ 0-\$ 99$

Allan J. Cigler - A

Caleb M. Clark - A
Potrick Coby

Michael J. Coppedge

Alex N. Dragnich - A

Pat M. Dunham - A

Cynthia Enloe - A

James W. Fesler - A

Timothy Fuller

Lawrence Graham - A

Martin Gruberg - B

Glen Halva-Neubauer

Roger Hamburg - A

Jonathan Hartlyn

Alice L. Hearst

Charles F. Hermann

Dean R. Hewitt

Erik P. Hoffmann - A

Susan E. Howell

Malcolm E. Jewell - A

Charlotte Joseph

Junko Kato

Herbert Kaufman - A

Richard C. Kearney - A

Anthony King - A

Henry Krisch - A

Jerome S. Legge, Jr. - A

Lance Leloup

Jonathan Lemco - A

Elaine S. Levine - A

Jeremy R.T. Lewis - A

Robert C. Lieberman - A

Charles H. Lipson

Lyceum Books, Inc. - A

Michael Margolis - A

Suzanne M. Marilley - C, B

Mark G. Mazzie - M

Clyde D. Mckee, Jr. - A

Richord L. Merritt - A

David R. Morgan - A

Stuart S. Nagel - A

Mark A. Peterson - A

Edward B. Portis - A

Wendy M. Rahn - A

Ronald B. Rapoport - S

Paul Rejai - A

Bernard H. Ross - A

Blair Ruble

Laura J. Scalia - A

Beth S. Schapiro

Henry B. Sirgo - A

Theda Skocpol - B

Robert J. Spitzer - A

Charles B. Turpin

Marvin G. Weinbaum - A

Gregory W. White

Janet L. Womack

Iris Marion Young
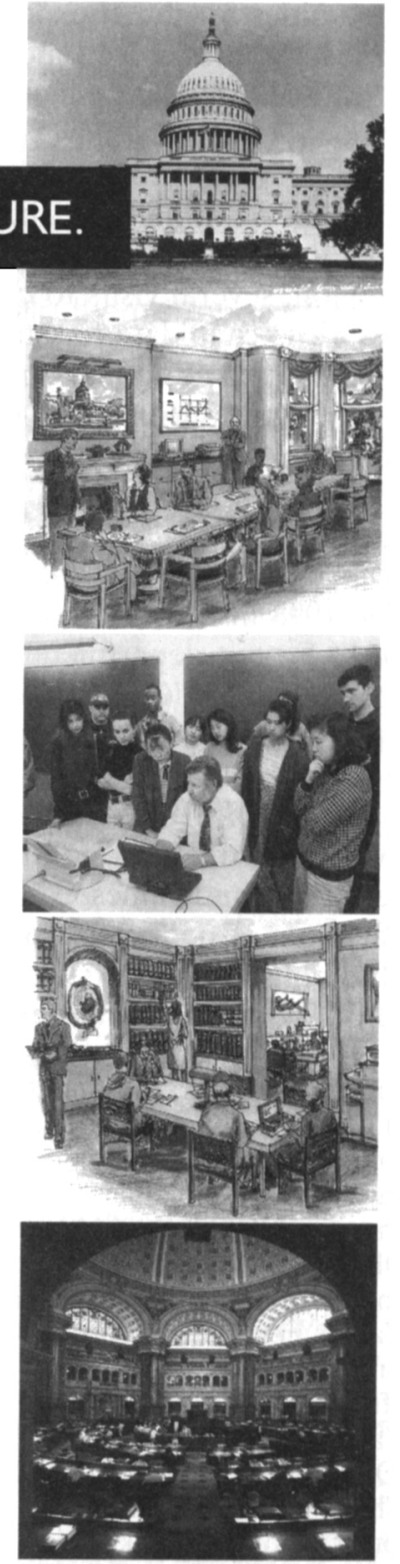

KEY

$C=$ General Compoign Fund, $A=$ Artinian Fund,

$B=$ Bornett Fund,

$\mathrm{Br}=\mathrm{Bryce}$ Fund

$M=$ Miller Fund

$P=$ Presidency Research Fund,

$W=$ Women \& Politics Fund 
trips designed to provide additional opportunities for direct exposure to American politics, government, history, and culture. These include a day spent viewing the government and public works projects of the City of Carbondale, a half day spent observing the federal district court for the region, several visits to campaign stops of the candidate for state senate who served as codirector, a very full day spent touring a not-forprofit hospital located in East St. Louis, Illinois, and a visit to the Fourth of July celebration and historical sites in a nearby small town.

The 1998 institute was directed by John L. Foster, department of political science at SIUC. Codirectors of the program were John S. Jackson, vice president for academic affairs and provost of SIUC; Barbara L. Brown, department of political science, SIUC; and James G. Leibert, department of political science,

Dickinson State University.

The 1998 participants were

Patricia Berrotaran, National University of Quilmes, Buenos Aries, Argentina

Jamil Hasanov, Higher Diplomatic College, Baku, Azerbaijan

Mohammed Abdul Wadud Bhuiyan, Dhaka University, Dhaka, Bangladesh

Fernando Lattman-Weltman, Getulio Vargas Foundation, Rio de Janeiro, Brazil

Wilfred N. Gabsa, University of Yaounde II, Yaounde, Cameroon

Li Qingsi, Renmin University, Beijing, China

Edourd Mpongo-Bokako, Universitie de Kinshasa, Kinshasa, Congo

Pavel Barsa, Masaryk University, Brno, Czech Republic

Hussein Jemma, Addis Ababa University, Addis Ababa, Ethiopia

Felix Kumah Godwin Anebo, University of Ghana, Legon, Ghana

Soja Madhavan, Sree Narayana College, Chempazhanthi, Thiruvananthapuram, India

Aleksius Jemadu, University of Parahyangan, Bandung, Indonesia

Shadrack Wanjala Nasong'o, University of Nairobi, Nairobi, Kenya

Ahmad Nizar Yaakub, University Malaysia Sarawak, Sarawak, Malaysia

Diana J. Mendoza, Ateneo de Manila University, Quezon City, Philippines

Roman Igorevich Kotov, Moscow State Linguistic University, Moscow, Russia

Thanyawat Rattanasak, Chiang Mai University, Chiang Mai, Thailand

Neo Richard Simutanyi, University of Zambia, Lusaka, Zambia

\section{Recent Contributors to APSA Awards and Programs}

\section{Anthony DeSales Affigne \\ Yvette M. Alex-Assensoh \\ Kristi Andersen \\ Paul E. Arnold \\ Q. Whitfield Ayres \\ William I. Bacchus \\ Robyn M. Bishop \\ Katya D. Bowers \\ Allan R. Brown \\ Carol Buckland \\ Michael P. Canning \\ K. Lynn Cates \\ Richard Cheney \\ Celia F. Cohen \\ Jeffrey E. Cohen \\ Leonard A. Cole \\ Pamelaj. Conover \\ David Corvette \\ Charles U. Daly \\ I. M. Destler \\ Bradley F. Dyke \\ Lee Epstein \\ Jo Freeman \\ Henry C. Galant \\ Irwin N. Gertzog \\ Esther S. Goldstein \\ Elliott Fiedler \\ Beth C. Fuchs \\ Eugene Hayunga \\ Mark J. Herbst \\ Paul S. Herrnson \\ Donald V. Hester}

John F. Hoadley

J. Woodford Howard, Jr.

Loch K. Johnson

Mark P. Jones

Francis]. Keenan

Stanley Kelley Jr.

Robert O. Keohane

Robert E. Lane

Logan A. Lee

Ann Chih Lin

Alice M. Litwinowicz

Robert Lorish

Barry Mahoney

Curtis M. Masiello

Donald J. Matthewson

David Mcllvain

Dalmas H. Nelson

Julie L. Novkov

Grady H. Nunn

Williarn L. Oakley

James L. O'Sullivan

Robert A. Pastor

Dianne Pinderhughes

John J. Pitney, Jr.

Lucian W. Pye

Leroy N. Rieselbach

Susan Carol Rogers

Raymond K. Rossiter

Catherine E. Rudder
Lloyd I. Rudolph
Jean Schroedel
H. P. Secher
Steven Seidman
Steven A. Shull
Roberta S. Sigel
Andrea Y. Simpson
Theda Skocpol
Frank J. Sorauf
John D. Sprague
R. V. Steffel
Alfred C. Stepan
David Swickard
Ross B. Talbot

Norman C. Thomas

Edward Thompson; III

James A. Thurber

Alvin B. Tillery, Jr.

Betty W. Trotter

Arturo A. Valenzuela Ali Velshi

Barbara A. Vobejda

David B. Walker

Stephen L. Wasby

Melissa S. Williams

David Wilsford

Thomas R Wolanin

Bonnie Smith Whyte

Mark Wynn

John E. Yang

Martynas A. Ycas

Charles E. Young

\section{Thank You!}

\section{Section News}

\section{Ecological and Transformational Politics}

The award for best paper at the 1997 meeting was presented to Kristen Hill Maher, University of California, Irvine, "Space, Race, Class, and Immigrant Labor: Latino Workers in Fortressed Middle-Class Communities."

The book award was shared by Theodore Becker and Richard Couto, eds., Teaching Democracy by Being Democratic (Praeger Publishers, 1997) and Roger Powers and William Vogele, eds., Protest, Power and Change: An Encyclopedia of
Nonviolent Action from ACT-UP to Women's Suffrage Garland Publishers, 1997).

\section{New Political Science}

Newsletter Editor and Secretary/

Treasurer

Carl Swidorski, History/Political

Science, The College of Saint

Rose, Albany, NY 12203; 518-458-

5325; swidorsc@rosnet.strose.edu

1999 Program Coordinator

Manfred Steger, Department of

Political Science, Illinois State

University, Schroeder Hall, Normal, IL 61790-4500;

Mbstege@ilstu.edu 